EGU2020-2153

EGU General Assembly 2020

(c) Author(s) 2020. This work is distributed under

the Creative Commons Attribution 4.0 License.

\title{
Numerical modeling of snow avalanche dynamics based on the Material Point Method
}

\author{
Xingyue $\mathbf{L i}^{1}$, Betty Sovilla ${ }^{2}$, Stephanie Wang ${ }^{3}$, Chenfanfu Jiang ${ }^{4}$, and Johan Gaume ${ }^{1,2}$ \\ ${ }^{1}$ School of Architecture, Civil and Environmental Engineering, Swiss Federal Institute of Technology, Lausanne, Switzerland \\ ${ }^{2}$ WSL Institute for Snow and Avalanche Research, SLF, Davos, Switzerland \\ ${ }^{3}$ Department of Mathematics, University of California, Los Angeles, USA \\ ${ }^{4}$ Computer and Information Science Department, University of Pennsylvania, Philadelphia, USA
}

Snow avalanches are one of the most dangerous and catastrophic hazards in mountainous regions, which cause fatalities and property losses. Understanding the dynamics of snow avalanches is essential for designing safe and optimised mitigation measures. This study presents numerical modeling of snow avalanche dynamics, based on the Material Point Method (MPM) and an elastoplastic constitutive model for porous cohesive materials. MPM is a hybrid EulerianLagrangian numerical method, which can simulate processes with large deformation, collisions and fractures. The elastoplastic model consists of an ellipsoid yield surface, a hardening law, and an associative flow rule. It enables us to capture the mixed-mode failure of snow including tensile, shear and compressive failure. Both ideal and real terrains are modeled in our study. By varying the properties of snow on the ideal slope, the model can reproduce four typical reported flow regimes, namely, cold shear, warm shear, warm plug and slab sliding regimes. In addition, surges and roll-waves are observed especially for flows in the transition from cold shear to warm shear regimes. The evolution of the avalanche front, the free surface shape and the velocity vertical profile show distinct characteristics for the different flow regimes. In addition to the snow properties, slope angle and path length are changed to investigate their effects on the maximum velocity, the run-out distance and the avalanche deposit height. The relation between the maximum velocity and the run-out distance obtained from our MPM simulations is analyzed along with data collected from literature. Furthermore, we benchmark the MPM model by simulating snow avalanches on real terrain. The evolution of the avalanche front position and velocity from the MPM simulations are quantitatively compared with the measurement data from past studies. 